Revista de Psicología de la PUCP. Vol. XIII. No 2. 1995

\title{
FACTORES PSICOSOCIALES ASOCIADOS AL USO DE BEBIDAS ALCOHOLICAS Y TABACO EN ADOLESCENTES TARDIOS DE UNA UNIVERSIDAD PRIVADA EN LIMA
}

\author{
Cecilia Chau Pérez-Aranibar ${ }^{1}$
}

\begin{abstract}
Esta investigación tiene como objetivos principales evaluar las características del uso de bebidas alcohólicas y de tabaco, y los factores psicosociales asociados, en adolescentes que cursan el primer año de estudios en una universidad privada en Lima. Los resultados muestran que, entre los varones, la tasa de prevalencia de vida y de uso actual es mayor para el alcohol, siendo la cerveza la más utilizada. El patrón de consumo de las sustancias evaluadas de tipo social y recreativo. En relación a los aspectos psicosociales se encontraron tabaco, y los abstinentes totales o no usuarios de ambas sustancias. De los 466 adolescentes encuestados, sólo nueve reportaron que nunca habían usado bebidas alcohólicas ni tabaco (abstinentes totales o no usuarios) y sólo un ocho por ciento de los encuestados reportó prevalencia de vida de sustancias psicoactivas ilícitas.

Palabras Claves: prevalencia de vida, uso actual, usuarios, no usuarios, abstinentes totales, drogas sociales, drogas legales, sustancias psicoactivas ilícitas.
\end{abstract}

Psychosocial factors associated with consumption of alcoholic beverages and tobacco in late adolescents from a private university in Lima

This paper main objectives are to evaluate the characteristics of tobbacco and alcoholic beverage consumption as well as associated psychosocial factors in 466 adolescent freshmen in a private university in Lima.

We found life prevalence and current consumption rates are higher for alcohol among male students, being beer the most consumed alcohol beverage than orher spirits. The pattern of consumption of these substances is social and recreational. With regard to psychosocial aspects, similarities were found between alcoholic beverages and tobacco consumers and nonconsumers or totaly abstemious ones (neither alcohol nor tobacco).

Only nine adolescentes, out of 466 , reported that they had never consumed alcoholic beverages nor tobacco (non-consumers) and eight percent of the sample reported illegal psychoactive susbstance live prevalence.

Keywords: life prevalence, current consumption, consumers, non-consumers, total abstemious ones, social drugs, legal drugs, illegal psychoactive substances.

1. Psicóloga Clínica licenciada en la Pontificia Universidad Católica del Perú. Actualmente se desempeña como asistente de docencia en dicha Universidad. 



\section{Introducción}

El fenómeno del uso y abuso de drogas ha adquirido tal magnitud que no sólo se ha convertido en un problema de salud prioritario en nuestra comunidad, sino también en una grave amenaza a la estabilidad de las instituciones políticas y jurídicas de nuestro país, por las implicancias del narcotráfico.

En el Perú, la problemática de las drogas ocupa el tercer lugar de importancia dentro de los problemas nacionales que afectan al país: el primer lugar lo ocupan los problemas económicos, seguidos por los ocasionados por la violencia y terrorismo (CEDRO, 1993).

Las investigaciones sobre consumo de sustancias psicoactivas llevadas a cabo en nuestro medio que abarcan a la población total, comenzaron a realizarse a finales de la década del 70 . En 1986, se realizó la primera investigación en el área urbana compuesta por ciudades de más de veinticinco mil habitantes (Jutkowitz, et al. 1987), y en 1988 se hizo una similar (Ferrando, 1990).

En el último estudio llevado a cabo por CEDRO (CEDRO, 1993) se observa un patrón de consumo que se caracteriza por el uso generalizado de bebidas alcohólicas (92\%), seguidas del tabaco (66\%).

Ferrando (1991) encontró que la prevalencia de vida (uso de una sustancia alguna vez en la vida de una persona) para el alcohol, entre los adolescentes de 15 y 17 años era de 61 por ciento y 89 por ciento entre los de 21 y 24 años. La prevalencia de vida para el tabaco, entre los adolescentes de 15 y 17 años, fue de 48 por ciento y entre los de 21 a 24 años 77 por ciento. 
La “oportunidad” de probar drogas está relacionada con la edad. Parece claro que existe una tendencia para la iniciación temprana por las generaciones más jóvenes en la población urbana del país (Ferrando, 1990; Lerner, 1991).

Las investigaciones sobre el fenómeno del uso de drogas en el Perú son, principalmente, de corte epidemiológico (Carbajal, et al. 1980; Jutkowitz et al., 1987; Ferrando, 1990; 1991; CEDRO, 1993) o realizadas en poblaciones escolares (Sotomayor, 1976; Huamán, Tueros y Villanueva, 1983; León, Ugarriza y Villanueva, 1989; Villanueva, 1989b) centrándose en el uso y abuso de sustancias psicoactivas sin considerar otros factores asociados. Es decir, que los estudios que se han realizado se han centrado en la población general (de 12 a 50 años) y en la adolescencia temprana o media, dejando de lado a los adolescentes tardíos. La investigación sobre el consumo de drogas lícitas e ilícitas en estudiantes de instrucción superior es inexistente.

Por ello, surgió el deseo de investigar y conocer sobre los diferentes aspectos de la vida de estos jóvenes y orientamos nuestro estudio hacia una población de adolescentes de una universidad privada, debido a que las investigaciones previas reportaron una alta incidencia de la prevalencia de uso de bebidas alcohólicas y tabaco entre jóvenes con mayor grado de instrucción. Al parecer habría una correlación directa y positiva de la ingestión de bebidas alcohólicas y del tabaco en todos los estratos sociales y niveles de instrucción (Ferrando, 1991).

\section{Adolescencia. Factores asociados al uso de alcohol y tabaco}

Se ha planteado que diversos factores intervienen en la iniciación, mantenimiento y la interrupción de la auto-administración de sustancias psicoactivas. Se asume que el uso de drogas es un fenómeno complejo y no se ha podido demostrar la existencia de una "causa" única. Para explicar porqué se empieza a consumir o por qué se mantiene el consumo, hay que considerar la interacción de los factores intrapersonales, interpersonales y situacionales (Organización Mundial de la Salud, 1973; Edwards, Arif y Hodgson, 1981; Villanueva, 1989a; Navarro, 1992; Schall, Kemen y Maltzman, 1992) en lugar de las de características patológicas o "desviadas" (Barnea, Teichman y Rahav, 1992). 
Vivimos en una sociedad que consume drogas, rodeados por imágenes y modelos de gente que las usa. Los avisos de televisión representan el uso de drogas en dos formas: algunas ligadas a la bebida asociada con diversión; otras vinculadas al uso de drogas (medicación) para el alivio. Ambas dan al espectador el mensaje que existe una droga para alterar el estado de ánimo del ser humano y que es aceptable usarla para ese propósito. La mayoría de adolescentes que experimentan con drogas o las usan socialmente no llegan a ser dependientes; desafortunadamente, otros sí (Milhorn, 1990).

Los adolescentes tienen muchas razones específicas para empezar a usar drogas: lo hacen por recreación, como una forma de ayuda en la socialización con sus pares y como un rito de pasaje a la adultez. Las prueban porque su uso representa una nueva experiencia y porque les produce placer. También lo hacen para rebelarse, en respuesta a un impulso y como una parte de su autoexploración. Además, las usan para afrontar problemas personales, para reducir estrés, para aliviar ansiedad, fatiga o aburrimiento.

La razón principal que los adolescentes dan para el uso actual de drogas es que los hace sentirse bien y que no experimentan consecuencias adversas (Thorne y DeBlassie, 1985; Milhorn, 1990; CEDRO, 1993).

La curiosidad es una característica del ser humano y muchos jóvenes desean probar ciertas drogas para determinar, por sí mismos, sus efectos. Como gran número de jóvenes empiezan por probar las drogas en grupo, sobre todo el alcohol, cigarrillos y marihuana, pueden observar que al tratar de satisfacer su curiosidad pueden también adquirir la sensación de "pertenecer" al grupo, de asumir independientemente la responsabilidad de sus propias acciones o ambas cosas. La primera oportunidad y las subsiguientes pueden estar más rclacionadas con la necesidad de ser aceptado como persona o con una percepción de independencia, que con la curiosidad. Estos factores asociados con los efectos farmacológicos y de otra naturaleza que producen las drogas, convierten a estos productos en atractivos para algunas de las personas que las prueban.

Los estudios con adolescentes han mostrado que el uso de alcohol y tabaco está relacionado a un número de factores psicosociales y demográficos. Además, han sido reportadas asociaciones importantes entre fumar, beber y el uso de drogas ilegales. A pesar de que se han hecho intentos 
para elaborar y refinar las perspectivas teóricas a través de pruebas empíricas, la mayor parte de la investigación en el uso de alcohol y tabaco entre adolescentes sólo tiene un sustento indirecto en la teoría postulada (Thorlindsson y Vilhjalmsson, 1991).

Siguiendo el modelo planteado por Villanueva (1989a); Barnea, Teichman y Rahav (1992) y McDonald y Towberman (1993), vamos a dividir los factores relacionados en: factores interpersonales, intrapersonales y situacionales o ambientales.

\section{Influencias interpersonales}

El consumo de drogas parece estar bastante asociado al fenómeno de grupo, dando como resultado que la relación entre el consumidor y sus grupos de referencia o a los cuales pertenece (en particular, la familia y los pares) sean considerados como variables críticas para el análisis del consumo de drogas (Otero, Mirón y Luengo, 1989).

Se ha encontrado que el uso de bebidas alcohólicas y cigarrillos en los adolescentes está positivamente asociado tanto con el nivel de consumo de alcohol y cigarrillos de sus pares y padres, como con la aprobación de los mismos hacia estas sustancias. Los padres y amigos que furnan o beben representan normas y actitudes favorables hacia el cigarrillo y el alcohol, lo hacen más rápidamente disponible o sirven como modelos (Bauman, Foshee y Haley, 1992; Barnea, Teichman y Rahav, 1992; Connolly, et al., 1992; Anderson y Henry, 1994).

Para resumir la literatura revisada en relación a las actitudes y conductas parentales hacia el uso de sustancias, es claro que existen relaciones positivas significativas entre el uso de sustancias por parte del adolescente y por parte de sus padres; entre una ausencia de normas parentales y el uso de éstas, entre una falta de consistencia en el cumplimiento de esas normas y una ausencia de cercanía en la relación padre-hijo (Denton y Kampfe, 1994). Las preguntas sobre cuáles de esas variables son más significativas y sobre el predominio del uso y normas de los pares comparado al uso y normas de los padres, están abiertas a cuestionamiento debido a los resultados conflictivos que existen en la literatura (Dielman et al., 1990-1991). 
Las primeras investigaciones mostraron que el uso de drogas entre adolescentes está fuertemente influenciado por la asociación con pares que también usan drogas (Hirschi, 1969; Kandel, 1980; Tudor, Peterson y Elifson, 1980; Kaplan, Martin y Robbins, 1984; Kandel, 1985; Oetting y Beauvais, 1987; Swadi y Zeitlin, 1988; Castro, et. al., 1988; Simons, Conger y Whitbeck, 1988; Swaim, et al., 1989; Teck-Hong, 1989; Wilks et al., 1989; Otero, Mirón y Luengo, 1989; Dielman, et al., 1990-1991; Adger, 1991; Iannotti y Bush, 1992; Dinges y Oettin, 1993).

El hallazgo más consistente y reproducible en la investigación de drogas es la fuerte relación entre el comportamiento de un individuo que las consume y el uso actual de los amigos, ya sea éste percibido por el adolescente o reportado por los amigos (Kandel, 1980; Dinges y Oetting, 1993). Existen factores relacionados con los pares que permiten predecir el consumo de alcohol y tabaco, aun cuando otros factores estén controlados.

El límite del uso de drogas en el grupo de pares, auto-reportes de su consumo y la tolerancia percibida acerca del uso, son todos sólidos predictores del inicio del adolescente en el consumo de alcohol, tabaco u otras drogas ilícitas. Esta conclusión permanece válida pero ignora la pregunta de causalidad, es decir, ¿los adolescentes toman porque los amigos lo hacen? 0 , ¿los adolescentes que beben busc a amigos bebedores? (Dielman et al., 1990-1991).

Además, investigaciones recientes han tratado la influencia del uso y abuso de los pares y sus normas respecto al consumo de sustancias, donde el "modelado"o "aprendizaje sacial" parece ser el mecanismo más importante que explica la influencia de los pares en la medida que el grupo de consumidores se comporta por refuerzo facilitando el consumo de alcohol, tabaco y otras drogas (Otero et al., 1989).

La influencia del grupo de pares es un fuerte motivador en el mecanismo de iniciación (Huba, Wingard y Bentler, 1979; Teck-Hong, 1989; Swadi y Zeitlin, 1988; Adger, 1991; Alberts, et al., 1992; Baer y Carney, 1993). Sin embargo, los padres son importantes para establecer la frecuencia y patrones de bebida (Swadi y Zeitlin, 1988). Otros autores revelan la importancia de la influencia de los amigos en la cantidad y frecuencia 
de consumo, tanto como en la iniciación y continuidad de los patrones de abuso (Baer y Carney, 1993).

En general, los altos niveles de uso (y riesgo para uso futuro) están más asociados con una fuerte afiliación con los pares que con los padres (Thorne y DeBlassie, 1985; Swaim, et. al., 1989; Coombs et al., 1991). Entre los estudios que se han conducido se ha encontrado:

- que la frecuencia con la que los amigos usan alcohol, cigarrillos o marihuana es un predictor significativo e independiente de lo que los propios estudiantes reportan acerca de su consumo,

- el reporte de los mejores amigos permite predecir significativamente el consumo de alcohol de los estudiantes (Dielman et al., 1990-1991),

- la aprobación del uso de alcohol y tabaco de los amigos, más que la de los padres, sería la que influye en el consumo de los jóvenes (Connolly et al., 1992).

\section{Factores Intrapersonales}

Segal (1985-86) (citado por Villanueva, 1989a) ha confirmado hallazgos previos, encontrando tres factores asociados que explican los patrones de uso en la población normal: reducción de la tensión, efecto de la droga y relación con los pares. El primero se refiere al alivio de la ansiedad o la tensión; alejarse de los problemas y debido a la cólera o frustración. El efecto de la droga se relaciona con la curiosidad por la experiencia, en términos de novedad y esto está vinculado con la "buisqueda de sensaciones".

Barnea, Teichman y Rahav (1992) incluyen dentro de las características de personalidad, ansiedad de estado y rasgo, estado de ánimo depresivo y "brisqueda de sensaciones". Estas características son consideradas como factores emocionales que llegan a ser más intensos durante la adolescencia. Se ha encontrado que altos niveles de depresión, ansiedad y baja autoestima están relacionados a una actitud positiva hacia las drogas y la voluntad de hacerlo (McDonald y Towberman, 1993).

Existen varios factores que juegan un rol importante en el malestar emocional de los adolescentes y ellos aprenden estrategias de afrontamiento 
al estrés y tensión, en parte, de los adultos. En ese sentido, existiría una relación entre las habilidades de afrontamiento modelada por los padres y las técnicas de afrontamiento empleadas por sus hijos adolescentes. Estos últimos aprenderán a usar alcohol, aislarse, orar o buscar soporte, observando la conducta de sus padres (Adger, 1991).

\section{Factores situacionales $y$ de contexto}

El "medio" es una fuente de información que juega un rol definitivo en el compromiso de los adolescentes en el uso de sustancias. La exageración, la irresponsabilidad reportada y los avisos representativos del uso de varias sustancias - sobre todo el alcohol y tabaco- como conductas de riesgo se ha correlacionado con el uso de las mismas por los adolescentes (Swadi y Zeitlin, 1988).

La revisión de la literatura indica que, de los factores sociales que afectan la iniciación y continuación del uso de sustancias por adolescentes, dos deberían ser siempre considerados:

- la disponibilidad de la sustancia en el ambiente

- la experimentación previa con drogas (Huba, Wingard y Bentler, 1980; Teichman et al., 1989).

La "oportunidad" está fuertemente relacionada a la edad. Thorne y DeBlassie (1985) plantean que, para la población norteamericana, ésta se presenta mayormente entre los 18 y 25 años. Los adultos que consumen drogas se refieren comúnmente a la adolescencia como el periodo donde probaron drogas por primera vez (Swadi y Zeitlin, 1988).

El lugar donde se bebe también influye en el consumo de alcohol. A medida que se ingresa a la adolescencia tardía se empieza a beber más fuera de casa y en presencia de amigos (Connolly et al., 1992; Beck y Lockhart, 1992).

Algunos investigadores han sugerido que los adolescentes que fuman o beben están más proclives que los que no fuman o beben, a comprometerse en otras "actividades desviadas". De acuerdo con estos investigadores, en los adolescentes que fuman y usan alcohol, coexisten otras "conductas desviadas" como parte de un "sindrome de problemas de con- 
ducta" (Barnes, 1984; Donovan y Jessor, 1985; Jessor y Jessor, 1987; Thorlindsson y Vilhjalmsson, 1991).

Sabemos que cualquier experiencia con drogas es un fenómeno complejo en la medida que implica la interacción de factores farmacológicos, tales como la dosis, vía de administración y respuesta del usuario; aunadas a las expectativas personales y socio-culturales.

El patrón de uso de sustancias puede ser acumulativo, debido a que los sujetos pueden ampliar su repertorio de uso a otras drogas y también puede ser secuencial, porque se inicia con drogas lícitas a drogas ilícitas a lo largo de los años. Se han reportado asociaciones positivas entre fumar, beber y el uso de drogas ilícitas (Thorlindsson y Vilhjalmsson, 1991). Esto apoya el concepto de que la secuencia se inicia con las drogas legales. Sin embargo, hay que señalar que el uso de una droga en particular no conduce necesariamente a otra a través de la secuencia, como por ejemplo, el paso de la marihuana a las drogas "fuertes" (Villanueva, 1989a).

La importancia de la variable "acceso a las drogas ilegales" prueba ser una de las variables fundamentales que afectan el consumo de drogas en adolescentes (Otero et al., 1989).

\section{Planteamiento del Problema}

La presente investigación tiene como objetivo general evaluar las características del consumo de bebidas alcohólicas y tabaco, así como los factores psicosociales relacionados a su uso, en adolescentes que cursan estudios superiores de primer año en una universidad privada en Lima.

Como objetivos específicos se proponen:

1. Caracterizar los aspectos demográficos significativos de una población que cursa los primeros años de estudios superiores en una Universidad Privada en Lima. Los factores a trabajar serán: datos personales, educacionales, económicos y desempeño académico.

2. Describir las características de uso de bebidas alcohólicas y tabaco de la población en referencia, en función de las siguientes áreas: prevalencia de vida, edad de primer uso, uso reciente, uso actual. 
3. Describir los aspectos psicosociales relevantes, tanto para los usuarios de bebidas alcohólicas y tabaco, como para los abstinentes totales de estas sustancias.

Los factores a trabajar serán:

a. Situación familiar: conformación del núcleo familiar, relación con los padres y hermanos

b. Relación con los amigos

c. Exposición a modelos como un factor vinculado al uso de bebidas alcohólicas y tabaco

d. Disponibilidad de bebidas alcohólicas y tabaco como un factor asociado al uso

Para el estudio se han definido los factores implicados, utilizando en todos los casos una definición operacional de medida.

\section{Metodología}

\section{Tipo de Estudio}

El estudio, siguiendo a Selltiz, et. al. (1980) es de tipo descriptivo, en la medida que la investigación tiene como objetivo describir a la población de adolescentes tardíos que cursan estudios superiores en una universidad privada en Lima, que usan alcohol y tabaco en lo que respecta a las características demográficas, de prevalencia y psicosociales.

\section{Muestra}

El universo de estudio estuvo constituído por 1373 alumnos de primer año, con un rango de edades de 16 a 20 años, matriculados en una universidad privada en Lima, del cual se obtuvo una muestra no aleatoria de 470 adolescentes. Finalmente, cuatro casos fueron excluídos por no cubrir con los requisitos de la investigación quedando la muestra en 466 casos.

Se utilizó un muestreo no probabilístico. Se trabajó por salones como unidades de muestreo y considerando el número de alumnos se seleccio- 
naron 12 aulas con mayor cantidad de estudiantes para garantizar la asistencia y lograr el tamaño de la muestra estimado.

\section{Instrumento}

El instrumento utilizado para la presente investigación fue la "Encuesta de los Factores Psicosociales Relacionados al Uso de Alcohol y Tabaco entre Adolescentes con Estudios Superiores", construída para fines de esta investigación.

El instrumento es una encuesta autoaplicable, que debe ser respondido en forma grupal y anónima.

Tres cuestionarios fueron tomados como modelos para la construcción del que se usaría en la investigación:

a. El Cuestionario sobre Uso de Drogas desarrollado por un equipo de trabajo del Ministerio de Educación (León, Ugarriza y Villanueva, 1989)

b. El Cuestionario para la Encuesta de Viviendas que aplicó en el Perú Ferrando en 1988 (Ferrando, 1990).

c. La Encuesta sobre "Aspectos Psicosociales del Uso de Sustancias Psicoactivas en Escolares de Lima Metropolitana", que mide aspectos intrapersonales e interpersonales de Villanueva (1993).

La versión definitiva de la encuesta quedó constituída por 117 preguntas correspondientes a tres grandes áreas: aspectos demográficos, aspectos psicosociales y prevalencia.

\section{Análisis Estadístico}

El análisis descriptivo y de frecuencias de la muestra se hizo por medio del programa estadístico SPSS PC+.

\section{Resultados}

A continuación se presentan los resultados del estudio de manera simultánea pará los usuarios de bebidas alcohólicas y tabaco. 
a) Prevalencia: a partir del Cuadro 1 podemos observar que la mayoría ha usado bebidas alcohólicas (b.a) (96.3\%) y tabaco (84.6\%) alguna vez en su vida, manteniéndose un porcentaje elevado en el uso actual, sobre todo para las bebidas alcohólicas (81\%).

La edad promedio en el inicio del uso es de 14 años para ambos casos.

En cuanto al sexo, en ambos casos la mayor parte son varones, en el caso de b.a $63.3 \%$ para la prevalencia de vida y $66.9 \%$ para el uso actual; y en caso del tabaco, $63 \%$ para la prevalencia de vida y $64.5 \%$ en el uso actual.

Cuadro 1: Prevalencia de uso de alcohol y tabaco (en porcentajes)*

\begin{tabular}{|l|lr|rr|ll|}
\hline \multirow{2}{*}{ Sustancia } & \multicolumn{2}{|c|}{$\begin{array}{c}\text { Usó alguna } \\
\text { vez en su vida }\end{array}$} & \multicolumn{2}{|c|}{ Uso reciente } & \multicolumn{2}{|c|}{ Uso actual } \\
\cline { 2 - 7 } & SI & NO & SI & NO & SI & NO \\
\hline Alcohol & 96.3 & $3.7 \mathrm{a}$ & 93.3 & $6.7 \mathrm{~b}$ & 81.0 & $19.0 \mathrm{c}$ \\
Tabaco & 84.6 & $15.4 \mathrm{~d}$ & 70.8 & $29.2 \mathrm{e}$ & 61.1 & $38.9 \mathrm{f}$ \\
\hline $\begin{array}{l}\text { * La lectura de este cuadro se realizará por filas. } \\
\text { Nota: total en base a uso alguna vez en la vida } \\
\mathrm{a}=458 ; \mathrm{b}=434 ; \mathrm{c}=432 \\
\mathrm{~d}=454 ; \mathrm{c}=370 \quad \mathrm{f}=373\end{array}$ \\
\hline
\end{tabular}

b) Características de uso la primera vez: la mayor parte usó bebidas acohólicas porque se las invitaron (60\%) y tabaco por curiosidad (80.8\%). En ambos casos fue facilitado por los amigos $(56.7 \%, 46.6 \%)$ y gran parte de ellos continuaron usando las sustancias $(90.8 \%$ y $66.2 \%$ ) (ver Cuadros 2 y 3).

- La bebida alcohólica más consumida es la cerveza seguida del vino y ron principalmente.

- La frecuencia de uso actual para la b.a es una vez por semana (40.9\%) o menos (44.3\%). En el caso del tabaco se tiende a usar diariamente más de una vez (36\%). 
Cuadro 2: Características del uso de bebidas alcohólicas por primera vez *

\begin{tabular}{|c|c|c|}
\hline Característica & F & $\%$ \\
\hline Usó bebidas alcohólicas porque le invitaron & $262 a$ & 60.1 \\
\hline Fue facilitado por los amigos & $246 \mathrm{~b}$ & 56.7 \\
\hline Continuó bebiendo & $395 \mathrm{c}$ & 90.8 \\
\hline \multicolumn{3}{|c|}{$\begin{array}{l}\text { * La lectura de este cuadro se realizará por filas. } \\
\text { Nota: } n=441 \text {. El número total de dígitos está en base a quienes usaron bebidas } \\
\text { alcohólicas alguna vez en su vida. } \\
\text { Los totales no son iguales porque se excluyen los casos de no respuesta: } \\
\quad a=436 ; b=384 ; c=435\end{array}$} \\
\hline
\end{tabular}

Cuadro 3: Características del uso de tabaco por primera vez *

\begin{tabular}{|lll|}
\hline Característica & F & $\%$ \\
\hline Usó tabaco por curiosidad & 308 a & 80.8 \\
Fue facilitado por los amigos & $177 \quad \mathrm{~b}$ & 46.6 \\
Continuó fumando & $251 \quad \mathrm{c}$ & 66.2 \\
\hline * La lectura de este cuadro se realizará por filas. \\
Nota: $\mathrm{n}=384$. El número total de dígitos está en base a quienes usaron tabaco alguna \\
vez en su vida. Los totales no son iguales porque se excluyen los casos de no respuesta: \\
$\quad \mathrm{a}=381 ; \mathrm{b}=384 ; \mathrm{c}=379$ \\
\hline
\end{tabular}

\section{Características del uso actual}

En cuanto a las b.a. la mayoría las usa para divertirse $(74.3 \%)$ y en un estado de ánimo alegre (58.7\%). En el caso del tabaco, fuma para no estar nervioso $(30.8 \%)$, y también se mencionó en segundo lugar porque le gusta (28\%), y en un estado de ánimo normal (43.3\%). En ambos casos, la mayor parte las usa antes o durante una fiesta $(77.2 \%, 71.4 \%$ ) (ver Cuadros 4 y 5 ).

El uso de bebidas acohólicas se asocia con el uso de tabaco y viceversa. El $50 \%$ que usa b.a. usa también cigarrillos y en porcentaje similar los usuarios de tabaco usan también b.a. La mitad de los que usaron b.a y 
tabaco alguna vez en su vida, continúan usando ambas sustancias en la actualidad. La mayor parte opina que usar b.a. no le genera problemas $(67 \%)$. De aquellos que opinan que sí, los problemas están relacionados consigo mismo $(71.6 \%)$.

Cuadro 4: Características del uso actual de bebidas alcohólicas*

\begin{tabular}{|lcc|}
\hline Característica & F & $\%$ \\
\hline $\begin{array}{l}\text { Usa bebidas alcohólicas para "vacilarse" } \\
\text { (divertirse) }\end{array}$ & $240 \mathrm{a}$ & 74.3 \\
Bebe cuando está alegre & $196 \mathrm{~b}$ & 58.7 \\
$\begin{array}{l}\text { Usa bebidas alcohólicas antes o durante } \\
\text { una fiesta }\end{array}$ & $260 \mathrm{c}$ & 77.2 \\
$\begin{array}{l}\text { Usa bebidas alcohólicas en la universidad } \\
\text { cuando está con los amigos }\end{array}$ & $19 \mathrm{~d}$ & 79.2 \\
\hline $\begin{array}{l}\text { * La lectura de este cuadro se realizará por filas. } \\
\text { Nota: } \mathrm{n}=350 . \text { Usuarios de bebidas alcohólicas. } \\
\text { Los totales no son todos iguales porque se excluyen los casos de no respuesta: } \\
\text { a=323; b=334; c=337; d=24 (en base a los que beben en la universidad). }\end{array}$ \\
\hline
\end{tabular}

Cuadro 5: Características del uso actual de tabaco*

\begin{tabular}{|lcc|}
\hline Característica & F & $\%$ \\
\hline Fuma para no estar nervioso & $58 \mathrm{a}$ & 30.3 \\
Fuma en estado de ánimo "normal" & $85 \mathrm{~b}$ & 43.4 \\
Fuma antes o durante una fiesta & $142 \mathrm{c}$ & 71.4 \\
Fuma en la universidad cuando está con amigos & $74 \mathrm{~d}$ & 78.7 \\
\hline (*) La lectura de este cuadro se realizará por filas. & \\
Nota: $\mathrm{n=228}$. El número total de digitos está en base a los usuarios actuales tabaco. \\
Los totales no son todos iguales porque se excluyen los casos que no respondieron: \\
a= 191; b=196; c= 199; d=94 (en base a los que fuman en la universidad). \\
\hline
\end{tabular}


En el caso del tabaco, se observa a diferencia del alcohol, que si bien el $58.9 \%$ afirma que no le genera problemas, el $41 \%$ afirma que sí, estando éstos relacionados consigo mismo (87.2\%). La mayoría de usuarios de b.a no ha intentado dejar de hacerlo. Sin embargo, la mayor parte de los usuarios de tabaco sí $(60 \%)$ siendo la razón principal por motivos de salud.

\section{Desempeño académico}

Tanto para los usuarios de b.a. y de tabaco, se presentan características similares: la mayor parte falta pocas veces a la universidad (55\%, 58.8\%), han repetido cursos por segunda o tercera vez $(86.2 \%, 88.6 \%)$; evalúan su desempeño $(60 \%, 58.8 \%)$ y calificaciones $(58.9 \%, 63.2 \%)$ de regulares, siendo su promedio ponderado entre 11 y $12(48.6 \%, 44.5 \%)$. No obstante, consideran que obtener buenas calificaciones es importante (44\%, $42.3 \%)$.

\section{Características de los amigos}

Para los usuarios de b.a. y tabaco se observan características similares: la mayoría tiene un grupo de amigos $(94 \%, 94.3 \%)$, estos grupos son mixtos y prefieren frecuentar a los de la universidad $(40.8 \%, 38.8 \%)$. La mayoría de usuarios tiene uno o más amigos íntimos. Entre las actividades que realizan con los amigos la de mayor frecuencia es ir a fiestas $(75.9 \%$, $77.1 \%)$, pasear $(61 \%, 59.5 \%)$, tomar licor $(56.2 \%, 57.7 \%)$.

Entre las actividades que realizan con los amigos la de mayor frecuencia es ir a fiestas $(75.9 \%, 77.1 \%)$, pasear $(61 \%, 59.5 \%)$, tomar licor $(56.2 \%$, $57.7 \%)$. En caso de los amigos íntimos se prioriza la conversación sobre sus problemas $(80.4 \%, 82.9 \%)$, dándose también las actividades antes mencionadas.

\section{Temas de conversación con amigos y/o amigos intimos}

En ambos casos, se observa que prefieren conversar sobre sus problemas personales $(89.9 \%, 88.4 \%)$, estudios $(68.1 \%, 71.3 \%)$, chicos/as $(68.7 \%$, $71.3 \%)$ y fiestas $(50.9 \%, 48.1 \%)$ cuando se reunen con los amigos íntimos. Una distribución para ambos casos se observa con los amigos, salvo que se prioriza el tema relacionado con las fiestas $(69.8 \%, 67.4 \%)$. 
Los encuestados perciben un adecuado vínculo con los amigos y/o amigos íntimos, se sienten apoyados, queridos y aceptados. Son los amigos con quienes tienen mayor confianza y con quienes se sienten mejor. Sin embargo, los padres son los que tienen mayor influencia en sus decisiones.

\section{En cuanto a las relaciones familiares}

En la relación entre sus padres, se observa que en términos generales es percibida como "buena". La mayor parte afirma que se llevan bien, discuten pocas veces, conversan entre ellos y con el hijo sobre sus intereses.

En cambio en la relación con la madre para los usuarios de b.a., si bien la mayoría considera que "siempre" es importante para su madre (75.4\%); en términos de la relación la mayor parte percibe que no los entienden $(46.5 \%)$ y que "pocas veces" conversan con ella sobre temas personales (45.3\%). Esto se refleja también en que "pocas veces" acuden a su madre cuando tienen un problema $(46.6 \%)$ o comparten sus inquietudes y/o deseos personales (45.7\%). En el caso de los usuarios de tabaco, las características son semejantes.

En la relación con el padre, en ambos casos, usuarios de b.a y tabaco, las características de la relación son semejantes a las de la madre. Si bien la mayoría considera que "siempre" es importante para su padre (69\%), por otro lado, perciben que no los entienden (44.4\%), y "pocas veces" conversan con él sobre temas personales $(51.4 \%)$. Así mismo, "pocas veces" acuden con él de tener un problema (55.6\%) o comparten sus inquietudes y/o deseos personales $(53.5 \%)$.

La percepción de la relación en la familia para ambos casos, es positiva en términos de que opinan que se llevan bien y muy bien.

Se aprecia al modelado en el uso de sustancias de sus familiares y pares. Tanto para los usuarios de bebidas alcohólicas como de tabaco, los amigos son quienes en su mayor parte usan b.a $(92.2 \%, 91.9 \%$ respectivamente), tabaco $(86.9 \%, 88.7 \%)$ y sustancias psicoactivas ilícitas $(38 \%, 41.9 \%)$.

En el caso de la pareja, la mayor parte usa alcohol $(50.4 \%, 48.1 \%)$ y tabaco $(44 \%, 52.5 \%)$, lo mismo con los hermanos $(56.5 \%, 54.6 \%)$. 
En relación al padre, se presenta en segundo lugar en cuanto a porcentaje de uso de b.a $(62.7 \%, 60.7 \%)$, también usan tabaco $(38.2 \%, 39.8 \%)$, pero en menor porcentaje si se compara con el uso de tabaco de los amigos y hermanos. En el caso de la madre, se reporta que no usa ninguna sustancia $(52.3 \%, 53.1 \%)$, y un porcentaje menor usa alcohol y tabaco $(30 \%)$.

Es importante señalar que para el caso de los amigos se reporta mayor incidencia del uso de sustancias ilícitas, siendo ésta en su mayor parte marihuana.

Por último, en relación a los abstinentes totales de bebidas alcohólicas y tabaco, debemos señalar que son sólo nueve casos y no se observan diferencias importantes en los aspectos psicosociales desarrollados.

\section{Discusión}

Es importante indicar que la muestra siendo no probabilística, sólo puede considerarse como representativa de los estudiantes de primer año de educación superior, de la universidad de donde fue extraída. Los resultados no pueden ser generalizables a los alumnos de primer año de otras universidades de Lima.

En relación al estrato social de procedencia y considerando las características socioeconómicas de la muestra, donde la mayoría de ellos proceden de colegios particulares, viven en zonas residenciales, sus padres tienen educación superior y dependen económicamente de ellos (Oficina de admisión de la universidad), podríamos asumir que es representativa de jóvenes que pertenecen a un estrato socioeconómico medio y medio alto.

En ese sentido la muestra coincide con las afirmaciones de Ferrando (1991), las que indican que el nivel educativo es diferenciado por estratos y tiene su mayor expresión en la clase alta donde casi el $60 \%$ ha aprobado algún año de instrucción superior o ha culminado su carrera, frente a un $30 \%$ en la clase media y un $20 \%$ en la baja.

En cuanto al consumo de las "drogas sociales", el estudio se centra en un grupo de adolescentes tardios, investigando la magnitud, forma de 178 
consumo y factores psicosociales asociados, sin buscar información sobre abuso ni dependencia, pues la literatura coincide en que uno de los indicadores primarios de aquellos es la pérdida de intereses y metas educacionales, así como su abandono (DSM III-R, APA, 1987; DSM IV, APA, 1994; ICD-10, WHO, 1989, Newcomb, et al., 1987).

Un factor que podría considerarse "influyente", dadas las características delicadas del tema, es la probabilidad de falseamiento u ocultamiento voluntario de la información de parte de los encuestados, debido en gran medida a la "deseabilidad social". Sin embargo, la técnica de aplicación de encuestas en grupo debilita esta posibilidad por el anonimato (Oetting y Beauvais, 1990).

En nuestro estudio encontramos una alta tasa de uso de bebidas alcohólicas y tabaco, siendo la prevalencia de vida y el uso actual mayor para el alcohol. Esto nos dice de la gran aceptación que tiene esta sustancia en el medio social, la cual se ve reforzada por las campańas publicitarias que promocionan directa e indirectamente su consumo, manifestándose en la elevada proporción de quienes han bebido en los últimos 30 días previos a la encuesta (81\%) o en el año anterior (93.3\%).

En cuanto al uso actual de tabaco, se da una situación similar. En las campañas publicitarias se asocian al uso de alcohol y tabaco, situaciones agradables y atractivas para los jóvenes, con contenidos vinculados a la diversión, alegría relaciones exitosas con el sexo opuesto y estatus. Además, la mayor parte de veces los personajes presentados en publicidad también son jóvenes, por lo cual es probable que se incremente la posibilidad de identificación de los televidentes adolescentes y se imite su comportamiento de consumo (Villanueva, 1989a; Atkin, 1990; Resnick, 1990).

La prevalencia de tabaco entre los adultos ha declinado durante los últimos años; sin embargo, entre adolescentes permanece alta y la edad de inicio es menor comparativamente a años anteriores. Esto último podría explicarse por el periodo de desarrollo en que se encuentran, en el cual las consecuencias a mediano y largo plazo no se evalúan con facilidad (Conrad, Flay y Hill, 1992).

En cuanto a las características de uso por primera vez, ambas sustancias fueron facilitadas principalmente por los amigos, lo cual corrobora lo ya 
planteado en otras investigaciones donde la influencia del grupo de pares es un fuerte motivador en el mecanismo de iniciación.

En relación a las características del uso actual, en su mayoría, la razón principal para el uso actual de ambas sustancias, es que los hace sentirse bien y que no experimentan consecuencias seriamente adversas en el presente.

El inicio temprano con una sustancia, según Newcomb et al. (1987), es sólo uno de los factores de riesgo para el abuso, y en nuestro estudio vemos que el hecho de estar en la universidad definitivamente señala que las aspiraciones educacionales son altas, por lo tanto no necesariamente llegarán al abuso o dependencia (Newcomb et al., 1987; Villanueva, 1989b).

Las drogas sociales, alcohol y tabaco, son ampliamente aceptadas y se consumen habitualmente por este grupo de jóvenes. Consideramos importante señalar que la mayoría de los que usan alcohol lo hacen como parte de su desenvolvimiento social, en circunstancias en que el consumo es parte del contexto cultural establecido en nuestro medio, a lo cual en parte se añadiría que no necesariamente se abuse de él; y a pesar que de todos aquellos que alguna vez en su vida han probado algún tipo de droga sólo unos cuantos terminan dependiendo de ella, todos los iniciados constituyen una población en riesgo y son un problema potencial que no se debe dejar pasar por alto.

En ese sentido, existe un elemento adicional: el adolescente que consume drogas legales a temprana edad, transgrediendo así una norma social o familiar, probablemente se le hará menos difícil probar drogas ilegales, sin que esto signifique caer en la generalización, es decir que quien fuma o bebe necesariamente va a consumir sustancias ilícitas.

Sin embargo, es un hecho comprobado que casi todos los que consumen sustancias psicoactivas ilegales, en el inicio hacia la dependencia a drogas, consumieron alcohol y/o tabaco (Kandel, 1975; Blaze-Temple y Kai-Lo, 1992).

Ambas conductas, son alternativas que adopta el adolescente en sus intentos de adaptación al medio en que vive, o su consumo es con la 180 
intención de tener un "contacto social" que por ejemplo, en el caso del alcohol se ve facilitado debido a su efecto desinhibidor, contribuyendo a su desenvolvimiento social (Freixa y Soler, 1981; Ferrando, 1990).

La sociedad actual es propicia para el desarrollo de este tipo de conductas, puesto que ofrece muy pocas alternativas al adolescente, especialmente en sus tiempos libres. Mientras que como ya se ha mencionado, los medios de comunicación, en especial la televisión, fomentan la imagen del joven fumando y bebiendo en su tiempo libre, asociando estas conductas con un alto estatus social y éxito con el sexo opuesto.

En este estudio se ha encontrado precisamente que las situaciones sociales conducen a un incremento en el consumo de tabaco y bebidas alcohólicas, asociando su consumo a la interacción con los amigos.

Una posible explicación para que no se reporte una alta incidencia de los problemas aquí discutidos es la juventud de la muestra analizada, pues se sabe que a medida que aumenta la edad, el consumo de bebidas alcohólicas y tabaco se incrementa y, por tanto, los problemas asociados al uso también.

No debemos dejar de lado que a pesar de ser legales y disponibles en nuestro medio, al fin y al cabo son sustancias psicoactivas, peligrosas para la salud física y mental del individuo y de su entorno.

Los usuarios de tabaco han manifestado su deseo de dejar de hacerlo, y la razón principal es por motivos de salud, por influencia de amistades y por decisión propia. Una probable explicación es que esto puede ser resultado de las campañas de prevención abiertas hacia el uso de tabaco, como por ejemplo, la frase "fumar puede ser peligroso para la salud" antes de los comerciales de televisión, o la toma de conciencia de los derechos de los "fumadores pasivos", que son las personas expuestas al humo de tabaco en el ambiente, el cual contiene mayores concentraciones de toxinas; además de las disposiciones gubernamentales al respecto y otras medidas como el "día del no fumador".

En relación a los aspectos psicosociales, encontramos que existe similitud entre las características psicosociales de ambos grupos: usuarios y no usuarios. 
A pesar de que los no usuarios no constituyen una muestra muy numerosa, creemos necesario analizarla, pues la investigación en drogas últimamente se está centrando no sólo en los usuarios, sino también en los perfiles psicosociales de los no usuarios.

Con respecto a los pares, se ha planteado que aquellos jóvenes que usan sustancias psicoactivas tienden a hablar regularmente sobre sus problemas personales con sus amigos, a diferencia de los que se abstienen de consumirlas, quienes tienen otras fuentes de soporte. Además, los que las utilizan participan con más frecuencia en actividades sociales (Otero et al., 1989; Coombs et al., 1991).

En nuestro estudio ambos grupos conversan regularmente de sus problemas personales con sus amigos, y los prefieren antes que a sus padres para tal fin. En cuanto a la participación en actividades sociales se corroboró lo antes mencionado.

Consideramos importante señalar que ambos grupos encuestados (usuarios y no usuarios) definen la relación con el padre y la madre de "amistad y confianza", lo cual no se refleja en la calidad de la relación.

Esto puede deberse a que dado el periodo adolescente que atraviesan, y por las características cognitivo-afectivas del ser humano al juzgar situaciones que lo implican íntimamente, se encuentra un cierto nivel de inconsistencia en este punto, señalando además que la percepción de ser importantes para sus padres también guarda relación con esto.

En nuestro estudio encontramos que los usuarios y los no usuarios se sienten más comprendidos por los amigos, y los padres son las personas que tienen más influencias sobre ellos, no encontrándose las diferencias planteadas en la literatura.

Una explicación probable podría ser que hemos evaluado las "drogas sociales" en función al uso, y las referencias anteriores están planteadas en función al abuso de éstas o al uso de sustancias psicoactivas ilícitas.

Es indiscutible entonces, que los padres tienen un papel constante como "personas de referencia", incluso en la adolescencia tardía, ofreciendo a sus hijos el aprendizaje y las experiencias que le permitan adaptarse al medio (Fierro, 1985; Horrocks, 1986). 
La Teoría del Aprendizaje Observacional (Bandura, 1977) sostiene que el uso de sustancias es aprendido por modelado e imitación. La conducta de beber y fumar, así como la aprobación o no del uso por parte de los amigos y de los padres, está asociado con las características del uso de los adolescentes (Wilks et al., 1989; Connolly et al.,1992; Van Roosmalen y McDaniel, 1992; Dinges y Oetting, 1993; Anderson y Henry, 1994; Denton y Kampfe, 1994).

Los amigos de los usuarios son quienes en su mayoría usan alcohol, tabaco y sustancias psicoactivas ilícitas (en ese orden), corroborando que la "conducta de consumo", así como el número de los pares que consumen, son los factores más importantes que afectan el uso de drogas en los adolescentes. Esto nos permite afirmar que si la mayor parte de los usuarios encuestados prefiere compartir su tiempo libre con los amigos, la posibilidad de incremento en el uso de las sustancias evaluadas es evidente.

El padre es la figura que, en segundo lugar, usa alcohol; y sabemos que el consumo de los hijos adolescentes incrementa si los padres consumen drogas legales e ilegales, ya que no son sólo modelos de imitación, sino que aumentan la probabilidad de que sus hijos internalicen la "legitimidad" de su consumo (McDemortt, 1984; Thorlindsson y Vilhjalmsson, 1991; Otero et al., 1989; Van Roosmalen y McDaniel, 1992; Anderson y Henry, 1994).

En relación a los no usuarios, se observan diferencias con los usuarios, ya que si bien los amigos son los que en su mayor parte consumen tabaco y bebidas alcohólicas, la pareja (enamorado/a), padres y hermanos, en su mayoría no consumen ninguna sustancia, lo que podría explicar en parte la condición de abstinentes totales.

Lo que planteamos es la relación entre el consumo individual y del entorno familiar y amical; encontrando una estrecha relación entre el uso de los usuarios con la de sus amigos y padres.

Por otro lado, es importante destacar que de los 466 adolescentes de la muestra, sólo 9 reportaron que nunca habían usado bebidas alcohólicas ni tabaco (no usuarios). 


\section{Conclusiones}

1. El patrón de consumo de estas sustancias es de tipo social-recreacional.

2. Sólo un ocho por ciento de los encuestados reportó prevalencia de vida de sustancias psicoactivas ilícitas, siendo en su mayoría varones y la sustancia utilizada marihuana.

3. El uso está relacionado con su "fácil acceso", debido a que son sustancias psicoactivas legales de venta libre, y los encuestados no asumen consecuencias adversas inmediatas en el uso.

4. La razón principal para usar tabaco por primera vez fue la "curiosidad" y para usar bebidas alcohólicas "porque se las invitaron", siendo ambas facilitadas por los amigos.

5. El tabaco se usa por motivaciones personales, relacionadas al estado de ánimo; mientras que el uso de bebidas alcohólicas está fuertemente asociado a motivaciones sociales.

6. La mayor parte de los encuestados que ha tratado de dejar de consumir sustancias han sido los usuarios de tabaco, teniendo, como razón fundamental, la salud física.

7. En relación a los aspectos psicosociales se encuentran similitudes en los tres grupos estudiados (usuarios de bebidas alcohólicas, tabaco y abstinentes totales o no usuarios).

8. El estatus de legalidad que poseen las "drogas sociales" así como su promoción y difusión por los medios de comunicación asociados a modelos jóvenes exitosos, influyen en el consumo de las mismas; marcando una diferencia sustancial con respecto al consumo de las ilícitas.

\section{Sugerencias y limitaciones}

1. Consideramos importante el desarrollo de investigaciones que permitan ampliar los alcances del presente estudio y conocer las características particulares sobre el "uso" de sustancias psicoactivas, las moti- 
vaciones internas para el consumo y las actitudes hacia las drogas; enfatizando el estudio sobre el "uso intenso" o "abuso" de las "drogas sociales" y de las sustancias psicoactivas ilícitas.

2. Se debe considerar, especialmente, la selección de las poblaciones de estudio, ya que esta problemática se presenta en diferentes estratos socio-económicos y etapas evolutivas de desarrollo; sin embargo el énfasis que se propone debe incluir a poblaciones de adolescentes tardios que cursan estudios pre-universitarios y superiores (universidades, institutos, escuelas técnicas, etc.), incidiendo en los factores psicosociales, de tal manera que sirvan de base para la elaboración de campañas de prevención para cada grupo en particular.

3. Como parte de los programas de prevención sería importante el desarrollo de talleres educativos, no necesariamente de información sobre drogas, sino de aquellos que incidan en los factores de salud mental: estabilidad emocional, alta autoestima, adecuadas redes de soporte social (pares), etc; teniendo en cuenta que la relación con los amigos, es uno de los factores que más influye ya sea positiva o negativamente en el consumo de drogas.

4. Es conveniente trabajar en programas preventivos con los profesores y padres de familia, enfatizando sus roles como modelos de conducta y en la transmisión de valores.

5. Creemos que urge la necesidad de abordar esta problemática desde una perspectiva interdisciplinaria, que permita a los profesionales de las distintas ramas del saber vinculadas al tema, aportar de acuerdo a su nivel de participación.

6. Es necesario incluir cursos sobre aspectos psicológicos del consumo de drogas, dentro de la currícula universitaria.

7. Se debe continuar con esta línea de investigación dentro de la universidad donde se realizó el estudio, en niveles o años superiores; realizando estudios comparativos y longitudinales sobre la estabilidad de los patrones de consumo de los estudiantes conforme avanzan en su desarrollo psicosocial y carrera universitaria. 
8. En el presente estudio debemos reconocer algunas limitaciones relativas, como por ejemplo el hecho de no haber aplicado simultáneamente la encuesta, por razones administrativas de la universidad; además la época en la que se realizó el estudio era un ciclo de verano, lo que hace que esta muestra represente un grupo heterogéneo con respecto a la población de los primeros años de la universidad.

\section{Referencias}

American Psychiatric Association (1987). Diagnostic and Statistical Manual of Mental Disorders. (3rd ed.rev.). Washington DC: American Psychiatric Association.

American Psychiatric Association (1994). Diagnostic and Statistical Manual of Mental Disorders. (4th ed.). Washington DC: American Psychiatric Association.

Adger, H. (1991). Problems of alcohol and other drug use and abuse in adolescentes. Journal of Adolescent Health Care. 12, 606-613.

Alberts, J., Hecht, M., Miller-Rassulo, M. y Krizek, R. (1992). The communicative process of drug resistance among high school students. Adolescence. 27(105), 203-226.

Anderson, A. y Henry, C. (1994). Family system characteristics and parental behaviors as predictors of adolescent substance use. Adolescence. 29 (114), 405-420.

Atkin, Ch. (1990). Effects of televised alcohol messages on teenage drinking patterns. Journal of Adolescent Health Care. 11, 10-24.

Baer, J. y Carney, M. (1993). Biases in the perceptions of the consequences of alcohol use among college students. Journal of Studies on Alcohol. 54, 54-60.

Bandura, A. (1977). Social Learning Theory. New Jersey: Prentice Hall. Barnea, Z., Teichman, M. y Rahav, G. (1992). Personality, cognitive, and interpersonal factors in adolescent substance use: a longitudinal test of an Integrative Model. Journal of Youth and Adolescence. 21 (2), 187199.

Barnes, G.M. (1984). Adolescent alcohol abuse and other problem behaviors: their relationships and common parental influences. Journal of Youth and Adolescence. 13(4), 329-348. 
Bauman, K., Foshee, V. y Haley, N. (1992). The interaction of sociological and biological factors in adolescent cigarette smoking. Addictive Behavior. 17, 459-467.

Beck, K. y Lockhart, S. (1992). A model of parental involvement in adolescent drinking and driving. Journal of Youth and Adolescence. 21(21), 35-52

Blaze-Temple, D. y Kai Lo, S. (1992). Stages of drug use; a community survey of Perth teenagers. British Journal of Addiction. 87, 215-225.

Carbajal, C., Jerí, R., Sánchez, C., Bravo, C. y Valdivia, L. (1980). Estudio Epidemiológico sobre Uso de Drogas en Lima. Revista Sanidad de las Fuerzas Policiales. 41, 1-38.

Castro F., Maddahian, E., Newcomb, M. y Bentler, P. (1988). Determinants of cigarette smoking among adolescentes. Brown University Digest of Addiction and Application. 7(3), 32-35.

Cedro (1993). Opiniones Sobre Drogas en el Perú Población Urbana. Monografía de Investigación No8. Lima: CEDRO.

Connolly, G., Caswell, S., Stewart, J. y Silva, P. (1992). Drinking context and other influences on the drinking of 15-year-old. New Zealanders. British Journal of Addiction. 87, 1029-1036.

Conrad, K., Flay, B. y Hill, D. (1992). Why children start smoking cigarettes: predictors on onset. British Journal of Addictions. 87, 17111724.

Coombs, R., Paulson, M. y Richardson, M. (1991). Peers vs. parental influences in substance use among hipanics and anglo children and adolescents. Journal of Youth and Adolescence. 20(1), 73-89.

Denton, R. y Kampfe, Ch. (1994). The relationship between family variables and adolescent substance abuse: a literature review. Adolescence. 29(114), 474-495

Dielman, T. E., Butchart, A. T., Shope, J. Y. y Miller, M. (1990-1991). Environmental correlates of adolescent substance use and misuse: implications for prevention programs. The International Journal of the Addictions. 25 (7A \& 8A), 855- 880 .

Dinges, M. y Oetting, E.R. (1993). Similarity in drug use patterns between adolescents and their friends. Adolescence. 28(110), 253-266.

Donovan, J. E. y Jessor, R. (1978). Adolescent problem drinking: psychosocial correlates in a national sample study. Journal of Studies on Alcohol. 39(9) 1506-1524. 
Edwards, G., Arif, A. y Hodgson, R. (1981). Nomeclature and Classification on Drug and Alcohol Related Problems: a WHO Memorandum. Bulletin of the World Health Organization. 59, 225-242.

Ferrando, D. (1990). Uso de Drogas en las Ciudades del Perú. Monografía de Investigación No. 5. Lima: CEDRO.

Ferrando, D. (1991). Los Jóvenes en el Perú. Opciones, Actitudes y Valores. Lima: CEDRO.

Fierro, A. (1985). Desarrollo Social y de la Personalidad en la adolescencia. En: Carretero, M; Palacios, J. y Marchesi, A. (1985). Psicología Evolutiva, Adolescencia, Madurez y Senectud. Madrid: Alianza Editorial.

Freixa, F. y Soleri, I. (1981). Toxicomanías. Un enfoque multidisciplinario. Madrid: Fontanella.

Hirschi, T. (1969). Causes of Delincuency. Berkeley, CA: University of CA press.

Horrocks, E. (1986). Psicologia del Adolescente. México: Trillas.

Huamán, J., Tueros, M. y Villanueva, M. (1983). Aspectos Psicosociales Relacionados con el Uso de Drogas en Adolescentes de Lima Metropolitana: Pontificia Universidad Católica del Perú. Lima: Inédito.

Huba, G. J., Wingard, J.A. y Bentler, P. M. (1979). Beginning adolescent drug use and peer and adult interaction patterns. Journal of Consulting and Clinical Psychology. 47, 265-276.

Huba, G. J. y Bentler, P. M. (1980). The role of peers and adults models for drug taking at different stages of adolescence. Journal of Youth and Adolescence. 9(5), 449-465.

Iannotti, R. y Bush, P. (1992). Perceived vs. actual friends use of alcohol, cigarettes, marijuana, and cocaine: wich has the most influence?. Journal of Youth and Adolescence. 21(3), 375-389.

Jessor, R. y Jessor, S. (1987). Problem Behavior and Psychosocial Development: a longitudinal study of youth. New York: Academic Press

Jutkowitz, J., Arellano, R., Castro De La Mata, R., Davis, P., Elinson, J., Jerí, R., Shaycoft, M. y Timaná, J. (1987). Uso y Abuso de Drogas en el Perú. Monografía de Investigación No. 1. Lima: CEDRO.

Kandel, D.B. (1975). Stages in adolescent drug use. Science. 190, 912-914. Kandel, D.B. (1980). Drug and drinking behavior among youth. Annual Review of Sociology. 6, 235-285. 
Kandel, D.B. (1985). On process of peer influence in adolescent drug abuse: a developmental perspective. Advances in Alcohol and Substance Abuse. 4, 139-163.

Kaplan, H., Martin, S. y Robbins, C. (1984). Pathways to adolescent drug use: self-derogation, peer influence, weakening of social controls and early substance use. Journal of Health and Social Behavior. 25, 270289.

León, F., Ugarriza, G. y Villanueva, M. (1989). La Iniciación y el Uso de Sustancias Psicoactivas: un Análisis de Datos en una Encuesta Nacional de Colegios. Lima: Ministerio de Educación, Convenio Perú-AID.

Lerner, R. (1991). Drugs in Peru. Reality and Representation. Nijmegen: Drukkerij.

McDermott, D. (1984). The relationship of parental drug use and parents attitude concerning adolescent drug use to adolescent drug use. Adolescence. 19(73), 89-97.

McDonald, M. y Towberman, D. (1993). Psychosocial correlates of adolescent drug involvement. Adolescence. 28(112), 925-936.

Milhorn T. (1990). Chemical Dependence. Diagnosis, Treatment, and Prevention. New York: Springer-Verlag.

Navarro, R. (1992). Cocaína. Aspectos Clínicos, Tratamiento y Rebabilitación. Lima: Libro Amigo.

Newcomb, M., Maddahian, E., Skager, R. y Bentler, P. (1987). Substance abuse and psychosocial risk factors among teenagers: association with sex, age, ethnicity and type of school. American Journal of Drug and Alcohol Abuse. 13(4), 413-433.

Newcomb, M.D., Fahy, B.N. y Skager, R. (1988). Correlates of cocaine use among adolescents. The Journal of Drug Issues. 18(3), 327-354.

Oetting, E. y Beauvais, F. (1987). Peer Cluster Theory, socialization characteristics and adolescent drug use: a path analysis. Journal of Consulting Psychology. 34, 205-213.

Oetring, E. y Beauvais, F. (1990). Adolescent drug use: findings of national and local surveys. Journal of Consulting and Clinical Psychology. 58(4), 385-394.

Organización Mundial de la Salud. Serie de informes técnicos (1973). La Juventud y las Drogas. Informe de un Grupo de Estudio de la OMS. Ginebra: OMS. 
Otero, J.M., Mirón, L. y Luengo, M. (1989). Influence of family and peer group on the use of drugs by adolescents. The International Journal of the Addictions. 24(11), 1065-1082.

Resnick, M. (1990). Study group report on the impact of televised drinking and alcohol advertising on youth. Journal of Adolescent Health Care. $11,25-30$.

Schall, M., Kemen, A. y Maltzman, I. (1992). Factors associated with alcohol use in university students. Journal of Studies on Alcohol. 53(2), 122-136.

Selltiz, C., Wrightsman, L. y Cook, S. (1980). Métodos de Investigación en las Relaciones Sociales. Madrid: Rialp.

Simons, R., Conger, R. y Whitbeck, L. (1988). A multistage social learning model of the influences of family and peers upon adolescentes substance abuse. The Journal of Drug Issues. 18(3), 293-315.

Sotomayor, J. (1976). El Problema de las Drogas en un Grupo de Estudiantes de Secundaria de Lima. En: Alarcón, R., Infante, J., Ponce, C. y Bibolini, A. (Eds). La Investigación Psicológica en el Perú. Lima: Sociedad Peruana de Psicología.

Swadi, H. y Zeitlin, H. (1988). Peer influence and adolescent substance abuse. a promising side? British Journal of Addiction. 83, 153-157.

Swaim, R., Oetting, E.R., Edwards, R.W. y Beauvais, F. (1989). Links from emotional distress to adolescent drug use: a path model. Journal of Consulting and Clinical Psychology. 57(2), 227-231.

Teck Hong, O. (1989). Peers as perceived by drug abusers in their drugseeking behaviour. British Journal of Addiction. 84, 631-637.

Teck Hong, O. y Israelowitz, R. (1989). Cross-cultural study of alcohol behavior among Singapore college students. British Journal of Addiction. 84(3), 319-321

Thorlindsson, T. y Vilhjalmsson, R. (1991). Factors related to cigarette smoking and alcohol use among adolescents. Adolescence. 26(102), 399-418.

Thorne, C.R. y Deblassie, R.R. (1985). Adolescent substance abuse. Adolescence. 20(78), 335-347.

Tudor, C.G., Peterson, D. y Elifson, K. (1980). An examination of the relationship between peer an parental influences and adolescent drug use. Adolescence. 15, 783-798. 
Van Roosmalen, E. y McDaniel, S. (1992). Adolescent smoking intentions: gender differences in peer context. Adolescence. 27(105), 87-105.

Villanueva, M. (1989a). Factores Psicológicos Asociados a la Iniciación y Habituación. En: León, F. y Castro de la Mata, R. (Ed.) (1989). Pasta Básica de Cocaina. Un estudio Multidisciplinario. Lima: CEDRO. Villanueva, M. (1989b). El Conocimiento de los Adolescentes sobre Drogas. Lima: Ministerio de Educación.

Villanueva, M. (1993). Factores Psicosociales asociados al uso de Sustancias Psicoactivas en escolares de Lima. (documento inédito).

Wilks, J., Callan, V. J. y Austin, D. (1989). Parent, peer and personal determinants of adolescent drinking. British Journal of Addiction. 84, 619-630.

World Health Organization (1989). Tenth Revision of the International Classification of Diseases Chapther V: Mental and Behavioral Disorders. Ginebra: WHO. 